International Journal of Power Electronics and Drive System (IJPEDS)

Vol. 10, No. 3, September 2019, pp. 1270 1280

ISSN: 2088-8694, DOI: 10.11591/ijpeds.v10.i3.pp1270-1280

1270

\title{
Single-bit modulator for wireless power transfer system
}

\author{
Dhafer J. Almakhles ${ }^{1}$, Akshya Swain ${ }^{2}$, Umashankar Subramaniam ${ }^{3}$ \\ ${ }^{1,3}$ Renewable Energy Lab, Communications and Networks Engineering, Prince Sultan University \\ ${ }^{2}$ Department of Electrical and Computer Engineering, University of Auckland
}

\begin{tabular}{|c|c|}
\hline Article Info & ABSTRACT \\
\hline Article history: & \multirow{12}{*}{$\begin{array}{l}\text { This paper proposes a single-bit ADC system based Proportional and Integral (PI) } \\
\text { controller to maintain a desired level of power transfer efficiency in Capacitive Power } \\
\text { Transfer (CPT) systems. In this paper, a simple single-bit ADC system i.e., Single- } \\
\text { Bit Modulator (SBM) is considered as an alternative to the commonly used multi-bit } \\
\text { ADC systems. Unique features of employing SBM are 1) its ability to convert analog } \\
\text { signals into single-bit signals and 2) its easy integrability in digital chips with linear } \\
\text { variable differential transformers (LVDTs) such as FPGAs. A SBM based PI (SBM- } \\
\text { PI) controller is designed to judicially interface with the single-bit output of SBM. } \\
\text { The proposed (SBM-PI) controller guarantees less hardware resources, latency and } \\
\text { regulates the output voltage to provide the desired power transfer efficiency. The be- } \\
\text { havior of SBM-PI controller is compared to that of a conventional multi-bit controller, } \\
\text { with the results of both controllers being identical. The effectiveness of the proposed } \\
\text { controller with SBM is further demonstrated using the experimental prototype of CPT } \\
\text { by implementing a SBM-PI controller using } 16 \mathrm{MHz} \text { ATmega8 microcontroller. The } \\
\text { experimental results from a laboratory prototype illustrate that SBM-PI controller suc- } \\
\text { cessfully regulates the output voltage of CPT to control the power flow. }\end{array}$} \\
\hline Received Nov 19,2018 & \\
\hline Revised Feb 23,2019 & \\
\hline Accepted Mar 15, 2019 & \\
\hline & \\
\hline Keywords: & \\
\hline Wireless power transfer & \\
\hline$(\mathrm{WPT})$ & \\
\hline Capacitive power transfer & \\
\hline (CPT) & \\
\hline Proportional integral (PI) & \\
\hline Single-bit Modulator & \\
\hline
\end{tabular}

Copyright (c) 2019 Institute of Advanced Engineering and Science. All rights reserved.

Corresponding Author:

Dhafer Almakhles,

Communications and Networks Engineering, Prince Sultan University,

P.O.Box No. 66833 Rafha Street, Riyadh 11586 Saudi Arabia.

Email: dalmakhles@psu.edu.sa

\section{INTRODUCTION}

Wireless power transfer (WPT) is a technique used to transmit power from a power source to a load without using physical wires or conductors. Electrical systems using WPT are becoming increasingly popular and have gained considerable attention in the research field for various applications including electric vehicle, biomedical implants, cellphones, contactless battery chargers etc. [1-3]. There are two distinct techniques of WPT, namely Inductive Power Transfer (IPT) and Capacitive Power Transfer (CPT). IPT uses the principle of electromagnetic coupling and is suitable for both low and high power applications, whereas CPT uses the principle of electric coupling and is suitable for lower power applications.

Among many advantages of CPT is that it has the ability to transfer power through metal barriers with high efficiency due to its resistance to any external interference [4-9]. However, one of the issues with WPT systems and CPT systems in particular is the inability to maintain the power flow at a desired level. This is mainly due to the uncertainties arise from load and circuit parameter variations and the operating frequency drifting of the power supply i.e., the operating frequency of the power inverter [10-14]. Hence, proportional-integral (PI) and proportional-integral-derivative (PID) controllers are often implemented to regulate the voltage in order to control power flow between the transmitters and receivers of WPT systems $[10,12,15,16]$. 
Conventional digital controllers manipulate discrete multi-bit signals in order to provide control to a particular system. A significant disadvantage of the conventional controller is the method in which the desired analog information is processed, sampled and converted into the discrete-time domain. Multi-bit analog to Digital Converters (ADC's) increase the complexity of hardware implementation and latency in order to accurately convey analog signals digitally. The hardware resources of the conventional multi-bit converters i.e., wires, input/output ports, etc., increase with resolution in order to maintain the high accuracy required in many control systems [17-21]. Multi-bit ADC's encompass additional limitations; such as the limitation of the finite word length of particular processors, setting further boundaries to the maximum available resolution and hardware resources. Furthermore, some multi-bit systems require a DAC which increases the required hardware even further.

As an effort to mitigate the demerits of large number of wires, complexity of implementations and maintenance, one technique known as Sigma-Bit Modulation (SBM), has become quite popular for achieving concise data transmissions and signal processing [22]. This technique uses SBM to convert the analog signal or multi-bit digital signal to single bit signal, which is known as single-bit signal, providing several advantages over the conventional control scheme and signal processing technique. First and the most obvious advantage of SBM is that the signal is represented by single bit, which simplifies transmission route to one wire per signal. Second, employing SBM reduces the hardware resources, complexity of implementation and remove the latency caused by the decimation filters in multi-bit ADC. Similar to discrete controllers with multi-bit inputs, discrete controllers with single-bit inputs manipulate discrete single-bit signals as opposed to multibit signals in order to provide control to a particular system. Single-bit controllers have the advantage of having the ability to use multiplexers as gains as opposed to the conventional multiplication gains. Thus, the processors with single-bit inputs are built simpler than conventional Nyquist rate processor due to the less usage of logic gates [18, 23, 24, 25, 26, 30]. The aforementioned features of single-bit control techniques make SBM is suitable many applications [30,31]. In particular, it becomes suitable to replace multi-bit ADC in power electronic systems controlled by high operating frequency; CPT is a prime example [28].

This paper proposes a SBM-PI controller to regulate the output voltage a CPT system. The effectiveness of the proposed control system will be examined by the regulating ability of a SBM-PI controller and comparing the performance against a conventional multi-bit controller executing the same task. The robustness of the controller will also be examined to fully identify the feasibility of a SBM based controllers on a WPT system.

The layout of this paper is as follows; Section 2 contains an overview of the CPT system to be controlled in this paper. Section 3 introduces the control problem, information about the single-bit signal, its generation, SBM and SBM-PI controller. Section 4 briefly covers the simulation and experimental results of CPT system controlled by SBM-PI controller. Finally, sections 5 and 6 will cover future recommendations and conclusions respectively.

\section{CAPACITIVE POWER TRANSFER}

This section encompasses the WPT system chosen to be controlled, i.e., a CPT. Briefly discussing the system, how it works, how it was designed and the response of the system.

\subsection{Overview}

Capacitive power transfer (CPT) systems take advantage of the principle of electric coupling in order to successfully maintain WPT. CPT system advantages include high voltage based efficiency, the ability to transfer between metal and low electromagnetic radiation. However, they are also associated with the disadvantages of being difficult to operate over large distances and having poor electric coupling safety which can be overcome with adequate insulation. The most critical disadvantage is the inability for a CPT system to naturally regulate their output voltage to a high degree of accuracy. Hence, a controller is implemented in order for accurate regulation of system output voltage. For a more in-depth discussion on CPT refer to [29].

For this paper, a simplistic CPT topology was chosen to allow for ease of analysis and is shown in Figure 1. As can be seen in the figure, the structure of the utilized CPT system includes a half-bridge inverter, an inductor $L$ in series with a capacitive coupling interface consisting of two pairs of coupling plates, and a full-bridge rectifier followed by a DC load. 


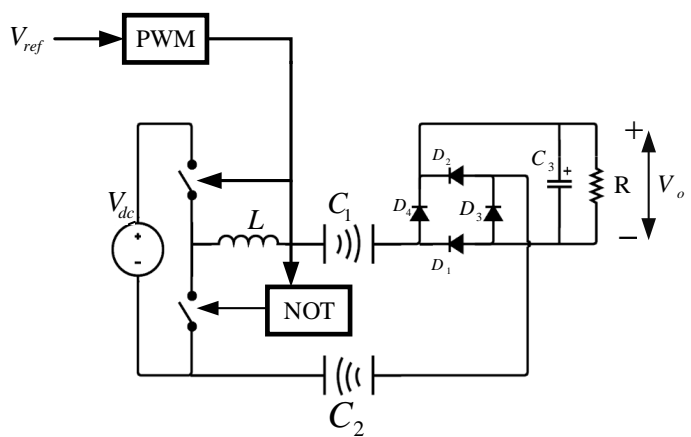

Figure 1. CPT system design

\subsection{Design principles}

In order to ensure the CPT is operating appropriately a few basic design principles must be followed. The first being that 'shoot-through' of the switches does not occur. Shoot-through occurs when the two switches are both turned on (closed) which effectively shorts the supply to ground. For simulations, in order to ensure this procedure does not occur the switches are controlled by a single PWM signal along with a simple NOT conductor. As for the hardware design, a half-bridge driver with a single input ensured that the switches never turned on simultaneously. This driver responded to a single PWM input signal and produced the appropriate signals which incorporated dead time to enable switches to successfully turn completely off.

The second design principle is to design for $L C$ resonance within the system. Resonance optimizes efficiency by matching the capacitor and inductor voltage magnitudes allowing for maximum power to the resistive load. The impedance of inductor and the two capacitors are opposite and directly related to frequency

$$
X_{L}=j \omega L, \quad X_{C}=\frac{1}{j \omega C / 2}
$$

where $X_{L}$ and $X_{C}$ are the inductor and capacitor impedance respectively, $\omega=2 \pi f$ where $f$ denotes the operating frequency of the half-bridge inverter, $L$ is the inductance and $C$ is the capacitance of each capacitor. Generally, the inductor value $L$ is chosen dependent on the system frequency $f$ and the capacitance $C$ of the electrically coupled metal plates. Due to the infinite reactance of capacitors at zero frequency, the two switches operating out of phase are required to act as a DC to AC inverter enabling current flow through the electrically coupled metal plates. The AC/DC rectifier then converts the signal back into DC for the load to consume.

\section{CONTROL SYSTEMS DESIGN}

This section encompasses the controller aspect of this paper, primarily designed to remove the error of the steady state value for applications that require precision in the power transferred. The section will cover the type of controller design and how the conventional controller is manipulated for single-bit based controller.

\subsection{Open loop control for CPT system}

The control scheme for the CPT systems is described as follow. A reference voltage $V_{\text {ref }}$ is used to determine the duty cycle of the PWM switches ranging from $0 \mathrm{~V}$ to $5 \mathrm{~V}$, representing $0 \%$ to $50 \%$ duty cycle, respectively. The output voltage $V_{o}$ is directly proportional to the duty cycle of the PWM signals controlling the DC to AC inverter. The maximum output voltage can be obtained when $V_{r e f}=2.5 \mathrm{~V}$ (or $50 \%$ duty cycle) and described as

$$
V_{o}=V_{d c} / 2 \gamma
$$

where $\gamma>1$ denotes uncertainties due to variation of operating frequency $f$, system parameters, distance between conductive plates, load changing in the CPT system, etc. The presence of the nonlinear components i.e., diodes and the uncertainties factors denoted by $\gamma$ in CPT often introduces high steady state error i.e.,

$$
e=V_{r e f}-V_{o}
$$


where CPT systems fail to accurately regulate voltage. Achieving exact or variable outputs for particular applications is difficult as the exact duty cycle required needs to be known. This behavior is undesired and attenuating steady state error is one of the main focuses for the upcoming controller where a PI is proposed in order to reduce the steady state error $e \approx 0$ along with the potential rise time.

\subsection{Single-Bit Modulator and Single-bit based controller}

This section encompasses the basics of the single-bit signal, briefly discussing its implementation and generation through the chosen modulation along with some of its advantages.

In terms of the single-bit based controller for a CPT, a single-bit signal is a method of representing an analog signal in the discrete time domain by using only one-bit of data per sample time. This signal consists of only two possible values; ' $h i g h$ - low' or 'on - off'; which interchanges between the two possible values at a relatively high switching frequency. The average value of the single-bit signal is equivalent to the average or root mean square (RMS) of the analog signal being replicated. Both the frequency and duty cycle are able to vary in order for the single-bit signal to accurately resemble the analog signal. Demonstrated in Figure 2 is the single-bit signal representation of a sine wave, with the sampling frequency reduced for clarity.

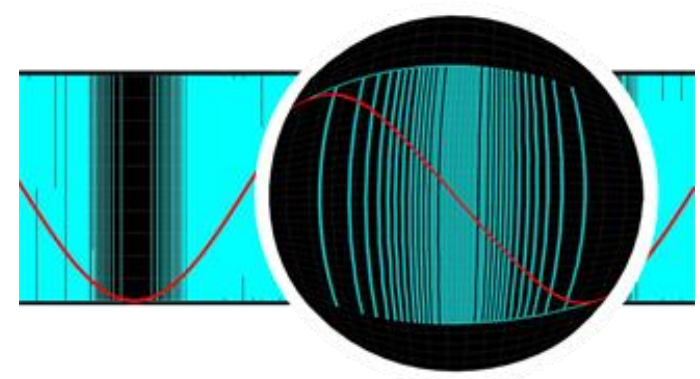

Figure 2. Single-bit representation of sine wave

\subsection{Single-Bit Modulator}

SBMs are type of single-bit ADCs where the input is an analog signal and the output is the corresponding digital single-bit signal. Basic SBMs are extremely simplistic, comprising of only a few components in comparison to multi-bit ADC. There are multiple topologies for producing a single-bit signal from an analog signal. Three possible techniques of SBM are delta $(\triangle-\mathrm{M})$, delta-sigma $(\Sigma \triangle$-M) or hybrid $\Sigma \triangle$-M. Cascading these techniques produces multi-order topologies of single-bit converters which enables an increase in the resolution of the digital signal. Figure 3 shows a simple SBM, which is used in this paper.

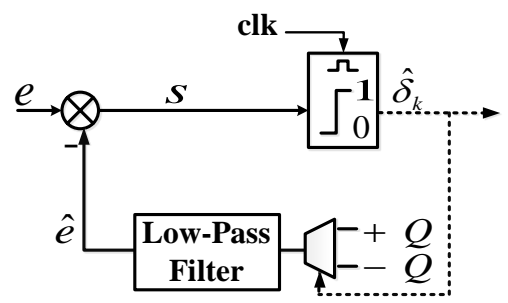

Figure 3. Single-bit modulator topology

This modulator comprises of a comparator, first order RC low-pass filter and a gain/multiplexer. Using a simple feedback network, this system attempts to pull the error between the comparators inputs to 0 , resulting in a single-bit signal representing that of the input analog signal. The output of the modulator is either 1 or 0 due to $\delta$ function, which is given by

$$
\delta_{k}=\frac{1}{2}(1+\operatorname{sgn}(s))= \begin{cases}1 & \forall s \geq 0 \\ 0 & \forall s<0\end{cases}
$$


since $\operatorname{sgn}(s)=\left\{\begin{array}{ll}+1 & \forall s \geq 0 \\ -1 & \forall s<0\end{array}\right.$ for all $t \in\left[k T_{s},(k+1) T_{s}\right]$ where $0<T_{s} \ll 1$ denotes the sampling period.

The input of the comparator is given by $s=e-\hat{e}$ where $e$ is defined in (1) and $\hat{e}$ denotes the average value (i.e., slow components) of the discontinuous function $\operatorname{sgn}(s)$ which is obtained by filtering it through first order low-pass filter

$$
\hat{e}:=Q \operatorname{sgn}(s)-\tau \dot{\hat{e}}
$$

where $\tau$ is the time constant and $Q$ is known as quantization boundary [26].

\subsection{Single-bit based controller}

The single-bit based controller can be classified to conventional PI controller and hybrid PI controller [26]. Implementing conventional single-bit PI controller is limited to which the proper $Q$ values used in SBMs of the proportional and the integral have to be carefully analyzed and selected in order to minimize the quantization noise. The SBM-PI controller which is used to control CPT system, is defined as

$$
\hat{u}=Q k_{p} \operatorname{sgn}(s)+k_{i} Q \int_{0}^{t} \operatorname{sgn}(s) d \tau
$$

where $\hat{u}$ is the control signal, $Q$ is the quantization boundary value of controller input $\mathrm{SBM}, k_{p}$ is proportional gain and $k_{i}$ is integral gain.

The requirement for the single-bit based controller is to have an input-output interface in singlebit format, which means the multi-bits arithmetic elements in the digital controller are replaced by single-bit function blocks. These blocks integrating SBM in arithmetic elements, such as adder, subtractor, and integrator, have been used and tested in different papers [26, 27].

In this paper, SBM based PI is implemented on microcontroller. Thus, it is of importance to convert the continuous-time system into its quasi-continuous approximation using the well-known Euler's discretization method. The discrete-time transformation of (4), which is shown in Figure 4, is given by $\hat{u}_{k}=\hat{u}_{k}^{p}+\hat{u}_{k}^{i}$ where

$$
\hat{u}_{k}^{p}= \begin{cases}K_{U p}=+Q k_{p} & \forall \hat{\delta}_{k}=1 \\ K_{L p}=-Q k_{p} & \forall \hat{\delta}_{k}=0\end{cases}
$$

and

$$
\hat{u}_{k}^{i}= \begin{cases}K_{U i} \rho^{-1} & \forall \hat{\delta}_{k}=1 \\ K_{L i} \rho^{-1} & \forall \hat{\delta}_{k}=0\end{cases}
$$

where $K_{U i}=Q T_{s} k_{i}, K_{L i}=-Q T_{s} k_{i}, T_{s}$ denotes the sampling frequency and $\rho=z-1$. The bold line is the multi-bit signal and the unbold line is the single-bits signal. These signal processing elements are detailed in [26].

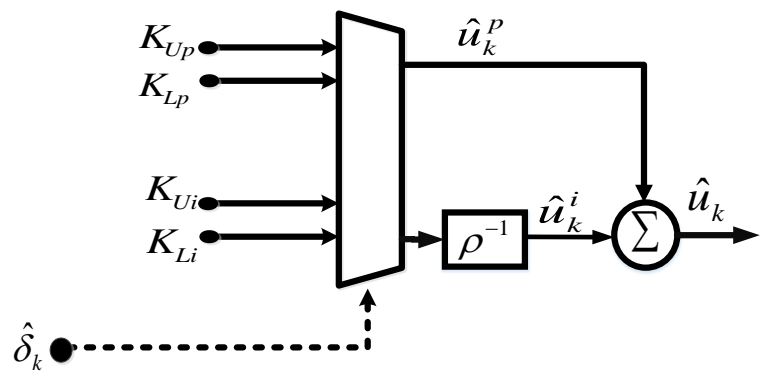

Figure 4. Hybrid single-bit PI 


\section{SIMULATION AND EXPERIMENTAL RESULTS}

Contained within this section are the findings of this paper. It demonstrates the performance and ability of the SBM-PI on a CPT system. Both simulation results and hardware results are presented.

\subsection{Hardware prototype and specifications of CPT system}

Multiple hardware prototypes were produced in the overall design process, with the final single-bit based control CPT system shown in Figure 5. The system parameters of CPT system, which is described by the equivalent circuit in Figure 1, are given in Table 1.

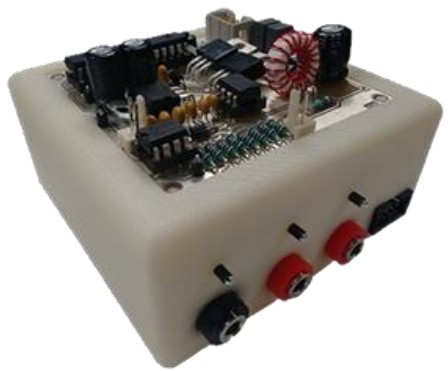

Figure 5. Prototype of the CPT system.

Table 1. Parameters of experimental prototype

\begin{tabular}{ll}
\hline Symbol & Value \\
\hline$V_{d c}$ & $12 \mathrm{~V}$ \\
$L$ & $27.6 \mu \mathrm{H}$ \\
$C_{1}$ & $470 n \mathrm{~F}$ \\
$C_{2}$ & $470 n \mathrm{~F}$ \\
$C_{3}$ & $470 \mu \mathrm{F}$ \\
$R$ & $47 \Omega$ \\
\hline & \\
\hline Controller & ATmega8 (max clk = 16MHz) \\
PWM's sampling frequency & $f_{s}=62.5 \mathrm{kHz}($ dead time of $0.5 \mu \mathrm{s})$ \\
half-bridge driver & IR2183 \\
MOSFETs $S_{1}$ and $S_{2}$ & IRF3205 \\
Diodes $D_{1}-D_{4}$ & $1 \mathrm{~N} 4001$ \\
\hline
\end{tabular}

\subsection{Simulation results}

Simulations were conducted in Matlab's simulink with the PLECS extension. Figure 6 (a) and (b) show both the simulated results as well as the hardware results for the open loop CPT system, with $3 \mathrm{~V}$ input resulting in a $4 \mathrm{~V}$ output. The system response has a steady-state error of approximately $1 \mathrm{~V}$ with an input of $3 V$ 's. By examining the issues pertained within the open-loop response of the CPT system the type of controller required to successfully achieve the desired response can be deduced. Although the system contained severe steady state error there was no oscillations residing in the transient of the open-loop response.

In order to gauge the success of the single-bit controller it was first produced in a simulated environment and compared to the performance of a simulated conventional multi-bit controller operating on the exact same system. Simulations provided sufficient evidence that single-bit based controllers provided accurate voltage regulating abilities. Figure 7 (a) shows the open loop system response to a $2 \mathrm{~V}$ and $4 \mathrm{~V}$ reference while Figure 7 (b) shows the controlled responses of the CPT to the same reference voltage, i.e., $2 \mathrm{~V}$ and $4 \mathrm{~V}$ reference using single-bit based PI controller with $k_{p}=255$ and $k_{i}=55$. As can be seen in Figure 7 (b), the controller was able to successfully eliminate the steady state value error present in the open loop responses in both cases for $2 \mathrm{~V}$ and $4 \mathrm{~V}$ input signals. Additionally, there was a decrease in both rise time and settling time for both scenarios. Furthermore, both the conventional and single-bit controllers responded exactly the same in simulations, this suggests that the single-bit controller performs at an equal level to that of the conventional controller. 

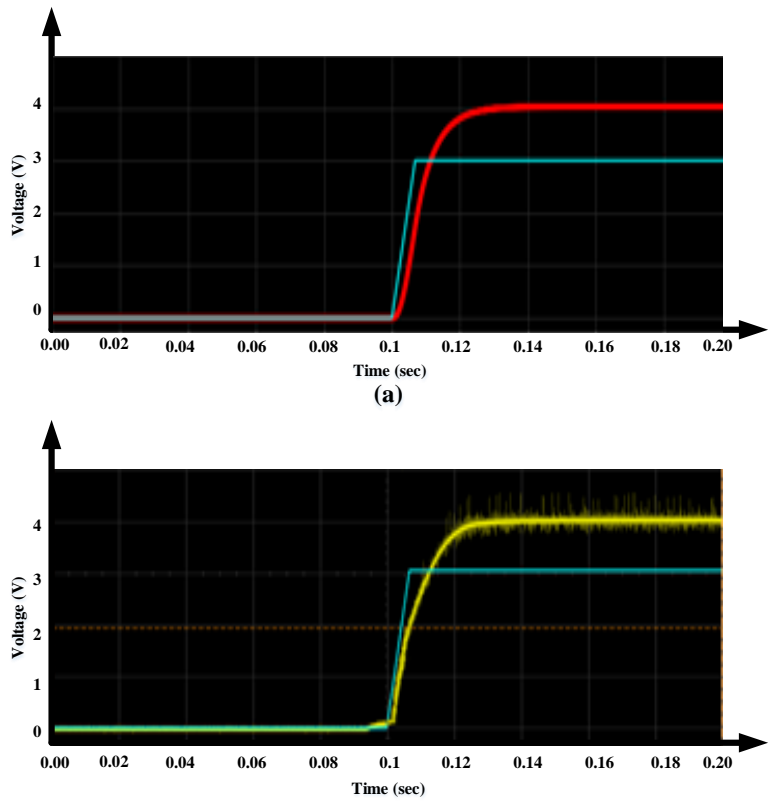

(b)

Figure 6. : Open loop CPT systems responce (a) Simulated resulted with $3 V$ input, and (b) Hardware response with $3 \mathrm{~V}$ input.

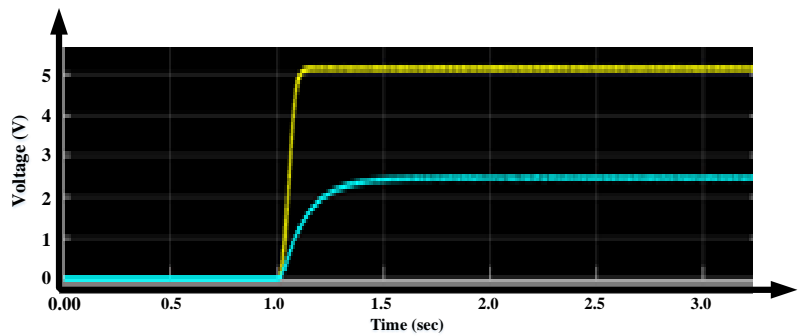

(a)

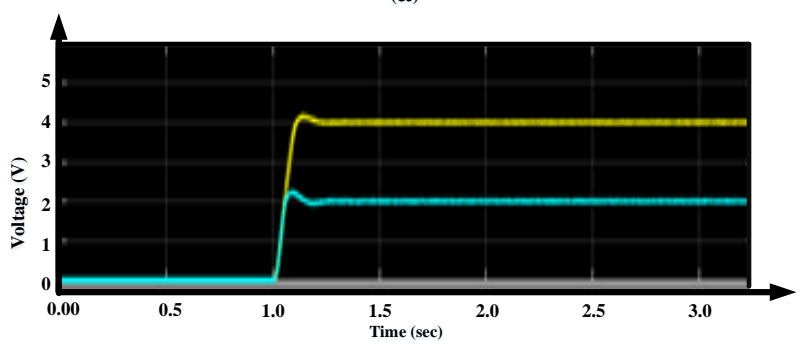

(b)

Figure 7. Simulation results for open and closed loop CPT systems responce (a) Simulated resulted for open-loop CPT systems with $2 V$ and $4 V$ inputs, and (b) Simulated resulted for controlled CPT systems with $2 \mathrm{~V}$ and $4 \mathrm{~V}$ inputs and PI controller.

\subsection{Experimental results}

Due to the success of the simulations on the CPT system, both a conventional and a single-bit controller were designed and implemented using hardware to prove the viability of the single-bit control methods even further. The responses obtained from the hardware resembled the simulations closely. They maintained 
similar overshoot, rise time and settling time along with obtaining the same response in terms of steady state voltage regulation. Demonstrated in Figure 8 is the controlled hardware response for a $2 \mathrm{~V}, 3 \mathrm{~V}$ and $4 \mathrm{~V}$ reference voltage. Finally, observing the robustness of the controllers was undertaken. A well performing controller is able to adequately regulate the response of a system even when system parameters are to venture away from their optimal states. Examples of parameters that could vary are the values of original component values, the DC supply, the operating frequency and the deterioration of components. The feature of a CPT system that is the most susceptible to change is the equivalent capacitance of the electrically coupled metal plates due to the potential air gap variances. The controller is able to successfully sustain the steady state voltage regulation with both a large increase and decrease in equivalent capacitance values. This suggests that the system is adequately robust.

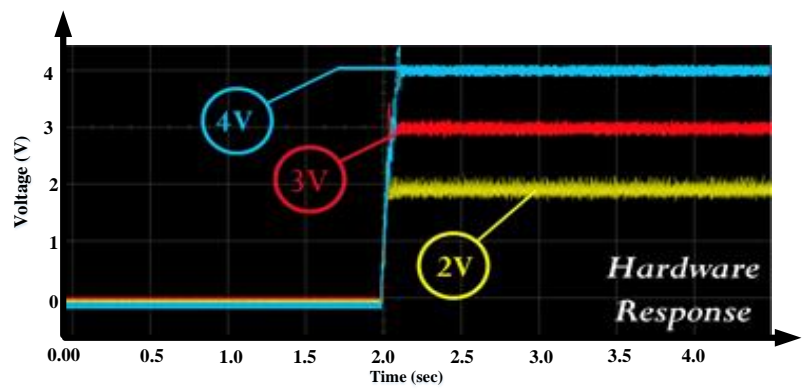

Figure 8. Controlled hardware response of the CPT system.

\section{FUTURE RECOMMENDATIONS}

One of the largest flaws in this paper was the accuracy of the single-bit signal. The technique used as a single-bit modulator was one of the simplest methods which reduced the overall accuracy slightly. However, the most significant issue was the ATmega8 microcontroller chosen. The ATmega8's limited clock speed was a severe hindrance. For future work in this field I would recommend using a processor with a larger clock speed. This improvement will result in a larger sampling frequency for the single-bit modulator which results in an increase of the analog to digital conversion resolution. Additionally, a multi-order delta-sigma modulator could be implemented in order to further increase the accuracy. The second flaw in this project is also related to the chosen microcontroller. The ATmega8's PWM feature, which is used to control the switches in the CPT system, was limited to $62.5 \mathrm{kHz}$ at 8-bits of accuracy which is much too low. Practical CPT Systems generally operate at higher frequencies in order to operate successfully with the low capacitance of electrically coupled metal plates. This issue would be rectified by using a faster processor and would allow for the controller to be implemented on a more practical system. Finally, implementing the single-bit controller in a networked control system. Networked control systems are becoming increasingly popular and single-bit is theorized to be a more viable option due to the benefits of transmitting single-bit as opposed to multi-bit packages.

\section{CONCLUSION}

In conclusion, a proposed method to manipulating a single-bit controller for a CPT system has been presented. This work included the design of both a conventional multi-bit controller and single-bit controller. The single-bit controller's performance was compared to that of the conventional multi-bit controller and the results demonstrated that both controllers were able to successfully regulate the response of the specified CPT system. This paper has proven the concept of using a single-bit controller on a CPT system and has provided headway for further research on the topic.

\section{ACKNOWLEDGEMENT}

The authors would like to thank both Nathan Pyle and Hossein Mehrabi for the help during the preparation of this work. 


\section{REFERENCES}

[1] J. T. Boys, G. A. Covic, and A. W. Green, "Stability and control of inductively coupled power transfer systems," IEE Proceedings - Electric Power Applications, vol. 147, no. 1, pp. 37-43, Jan 2000.

[2] A. P. Hu, C. Liu, and H. L. Li, "A novel contactless battery charging system for soccer playing robot," in Mechatronics and Machine Vision in Practice, 2008. M2VIP 2008. 15th International Conference on, Dec 2008, pp. 646-650.

[3] K. V. T. Piipponen, R. Sepponen, and P. Eskelinen, "A biosignal instrumentation system using capacitive coupling for power and signal isolation," IEEE Transactions on Biomedical Engineering, vol. 54, no. 10, pp. 1822-1828, Oct 2007.

[4] C. W. V. Neste, R. Hull, T. Abraham, J. E. Hawk, A. Phani, and T. Thundat, "Wireless single contact power delivery," in Wireless Power Transfer Conference (WPTC), 2015 IEEE, May 2015, pp. 1-4.

[5] C. Liu, A. P. Hu, and N. K. C. Nair, "Coupling study of a rotary capacitive power transfer system," in Industrial Technology, 2009. ICIT 2009. IEEE International Conference on, Feb 2009, pp. 1-6.

[6] N. Jamal, S. Saat, Y. Yusmarnita, T. Zaid, A. A. M. Isa, "Investigation on capacitor Compensation Topologies effects of Different Inductive Coupling Links Configurations," International Journal of Power Electronics and Drive System (IJPEDS), vo. 6, no. 2, pp. 274-281, 2015.

[7] A. Swain, D. Almakhles, M. J. Neath, and A. Nasiri, "Robust control of wireless power transfer system," in 2017 13th IEEE International Conference on Control Automation (ICCA), July 2017, pp. 1060-1065.

[8] F. K. A. Rahman, S. Saat, Y. Yusop, H. Husin and Y. Aziz, "Design and Analysis of capacitive Power Transfer System with and without Impedance Matching," International Journal of Power Electronics and Drive System (IJPEDS), vol. 8, no. 3, pp. 1260-1273, 2017.

[9] N. Nabila, S. Saat, Y. Yusop, M. S. M. Isa, A. A. Basari, "The design of auto-tuning capacitive power transfer for rotary applications using phased-locked-loop," International Journal of Power Electronics and Drive System (IJPEDS), vol. 10, no. 1., pp. 307-318, 2019.

[10] M. J. Neath, A. K. Swain, U. K. Madawala, and D. J. Thrimawithana, "An optimal pid controller for a bidirectional inductive power transfer system using multiobjective genetic algorithm," IEEE Transactions on Power Electronics, vol. 29, no. 3, pp. 1523-1531, March 2014.

[11] A. Swain, D. Almakhles, M. J. Neath, and A. Nasiri, "Robust h output feedback control of bidirectional inductive power transfer systems," Archives of Control Sciences, vol. 27, no. 1, 2017.

[12] J. U. W. Hsu, A. P. Hu, and A. Swain, "A wireless power pickup based on directional tuning control of magnetic amplifier," IEEE Transactions on Industrial Electronics, vol. 56, no. 7, pp. 2771-2781, July 2009.

[13] K. I. Woo, H. S. Park, Y. H. Cho, and K. H. Kim, "Contactless energy transmission system for linear servo motor," IEEE Transactions on Magnetics, vol. 41, no. 5, pp. 1596-1599, May 2005.

[14] P. Sergeant and A. V. D. Bossche, "Inductive coupler for contactless power transmission," IET Electric Power Applications, vol. 2, no. 1, pp. 1-7, Jan 2008.

[15] U. K. Madawala, M. Neath, and D. J. Thrimawithana, "A power-frequency controller for bidirectional inductive power transfer systems," IEEE Transactions on Industrial Electronics, vol. 60, no. 1, pp. 310317, Jan 2013.

[16] J. P. C. Smeets, T. T. Overboom, J. W. Jansen, and E. A. Lomonova, "Comparison of position-independent contactless energy transfer systems," IEEE Transactions on Power Electronics, vol. 28, no. 4, pp. 20592067, April 2013.

[17] T. Li and Y. Fujimoto, "Control system with high-speed and real-time communication links," IEEE Transactions on Industrial Electronics, vol. 55, no. 4, pp. 1548-1557, April 2008.

[18] X. Wu, V. A. Chouliaras, J. L. Nunez-Yanez, and R. M. Goodall, "A novel $\Delta \Sigma$ control system processor and its VLSI implementation," IEEE Transactions on Very Large Scale Integration (VLSI) Systems, vol. 16, no. 3, pp. 217-228, March 2008.

[19] D. Al-Makhles, N. Patel, and A. Swain, "Conventional and hybrid bit-stream in real-time system," in Intelligent Solutions in Embedded Systems (WISES), 2013 Proceedings of the 11th Workshop on, 2013, pp. 1-6.

[20] - "Bit-stream control system: Stability and experimental application," in Applied Electronics (AE), 2013 International Conference on, Sept 2013, pp. 1-6.

[21] — "A two-loop linear control utilizing $\Delta \Sigma$ modulator," in Intelligent Solutions in Embedded Systems (WISES), 2013 Proceedings of the 11th Workshop on, 2013, pp. 1-6. 
[22] D. Almakhles, A. K. Swain, and A. Nasiri, "The dynamic behaviour of data-driven $\Delta-\mathrm{M}$ and $\Delta \Sigma-\mathrm{M}$ in sliding mode control," International Journal of Control, vol. 90, no. 11, pp. 2406-2414, 2017. [Online]. Available:http://dx.doi.org/10.1080/00207179.2016.1250160

[23] D. Almakhles, A. K. Swain, A. Nasiri, and N. Patel, "An adaptive two-level quantizer for networked control systems," IEEE Transactions on Control Systems Technology, vol. 25, no. 3, pp. 1084-1091, May 2017.

[24] D. Al-Makhles, A. Swain, and N. Patel, "Delta-sigma based bit-stream controller for a d.c. motor," in TENCON 2012 - 2012 IEEE Region 10 Conference, nov. 2012, pp. 1 -5.

[25] D. Al-Makhles, A. K. Swain, and N. Patel, "Adaptive quantizer for networked control system," in European Control Conference (ECC), 2014, June 2014, pp. 1404-1409.

[26] D. J. Almakhles, A. K. Swain, and N. D. Patel, "Stability and performance analysis of bit-stream-based feedback control systems," IEEE Transactions on Industrial Electronics, vol. 62, no. 7, pp. 4319-4327, July 2015.

[27] N. Patel, S. Nguang, G. Coghill, and A. Swain, "Online implementation of servo controllers using bitstreams," in TENCON 20052005 IEEE Region 10, Nov 2005, pp. 1-6.

[28] J. Dai and D. C. Ludois, "A survey of wireless power transfer and a critical comparison of inductive and capacitive coupling for small gap applications," IEEE Transactions on Power Electronics, vol. 30, no. 11, pp. 6017-6029, Nov 2015.

[29] M. Kline, "Capacitive power transfer," Master's thesis, EECS Department, University of California, Berkeley, Dec 2010.

[30] _ _ "Sliding mode control as binary-based quantizers," Asian Journal of Control, vol. 0, no. 0. [Online]. Available: https://onlinelibrary.wiley.com/doi/abs/10.1002/asjc.2052

[31] D. Almakhles, "Two-level quantised control systems: sliding-mode approach," International Journal of Control, vol. 0, no. 0, pp. 1-9, 2018. [Online]. Available: https://doi.org/10.1080/00207179.2018.1484569

\section{BIOGRAPHIES OF AUTHORS}

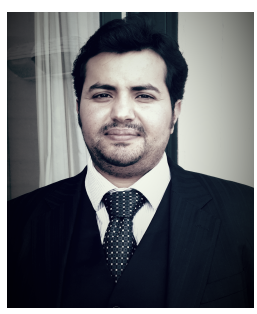

Dhafer Almakhles received B.E. degree in Electrical Engineering from King Fahd University of Petroleum and Minerals, Dhahran, Saudi Arabia in 2006 and completed his Masters degree (Hons.) and PhD from The University of Auckland, New Zealand in 2011 and 2016, respectively. Since 2016, he has been with Prince Sultan University - Saudi Arabia, where he is currently an assistant professor with the department of Communications and Networks Engineering and the Director of Science and Technology Unit. He has authored more than twenty published papers in the area of control systems. He served as a reviewer for many journals including IEEE Transactions on: Fuzzy Systems, Control of Network Systems, Industrial Electronics, Control Systems Technology as well as IEEE Control Systems Letters and International Journal of Control. His research interests include the hardware implementation of control theory, signal processing, networked control systems and sliding mode.

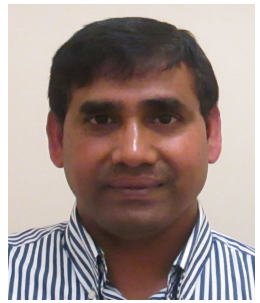

Akshya Kumar received the B.Sc. degree in electrical engineering and the M.Sc. degree in electronic systems and communication from the Sambalpur University, Sambalpur, India, in 1985 and 1988, respectively, and the Ph.D. degree from the Department of Automatic Control and Systems Engineering, University of Sheffield, Sheffield, U.K., in 1997.,From 1994 to 1996, he was a Commonwealth Scholar in the United Kingdom. Since September 2002, he has been with the Department of Electrical and Computer Engineering, The University of Auckland, Auckland, New Zealand. His present research interests include nonlinear system identification and control, biomedical signal processing, sensor networks, and control applications to power system and wireless power transfer systems.,Dr. Swain is an Associate Editor of the IEEE Sensors Journal. He is a Member of the Editorial Board of the International Journal of Automation and Control and the International Journal of Sensors, Wireless Communications and Control. 


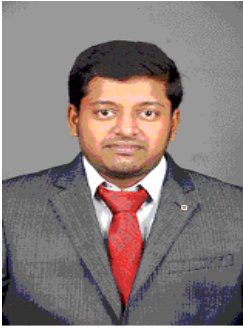

Umashankar Subramaniam is working as Associate Professor in Renewable Energy Lab, College of Engineering, Prince Sultan University, Saudi Arabia has 15+ years of teaching, research and industrial R and D experience. Previously, he worked as Associate Professor and Head, VIT Vellore as well as Senior R and D and Senior Application Engineer in the field of power electronics, renewable Energy and electrical drives. He is a Senior member-IEEE, and Member of IACSIT, IDES and ISTE. He has taken charge as Vice Chair - IEEE Madras Section and Chair IEEE Student Activities from 2018. He was executive member (2014-16) and Vice Chair of IEEE MAS Young Professional from 2017 by IEEE Madras Section. He has published more than 250+ research papers in national and international journals and conferences. He has also authored/coauthored/contributed 12 books/chapters and 12 technical articles on power electronics applications in renewable energy and allied areas. He is an Editor of Heliyon, an Elsevier journal. He received Danfoss Innovator Award-Mentor during 2014-15 and 2017-18, Research Award from VIT University during 2013-18. Also he received the INAE Summer Research Fellowship for the year 2014. Under his guidance 24 P.G students and more than 25 U.G Students completed the senior design project work. Also $6 \mathrm{PhD}$ scholars completed Doctoral thesis as Research Associate. He is also involved in collaborative research projects with various international and national level organizations and research institutions. 